\title{
3D follow-up study of facial asymmetry after developmental
}

\section{dysplasia of the hip}

\section{Abstract}

Objectives - To evaluate the change in facial asymmetry among subjects treated for developmental dysplasia of the hip (DDH) from childhood to adolescence.

Setting and Sample Population - A total of 39 adolescents (26 females and 13 males), born and treated for DDH during 1997-2001, participated in the first examination in 2007 (T1; at the age of 8.2) and in the follow-up in 2016 (T2; at the age of 16.6).

Material and Methods - In this longitudinal study, three-dimensional (3D) images were taken using a 3DMD face system based on a stereophotogrammetric method. Facial asymmetry was determined as the average distance $(\mathrm{mm})$ calculated between the original and superimposed mirrored face and the symmetry percentage $(\%)$ calculated as the face area where the distance between the original face and the mirrored surface does not exceed $0.5 \mathrm{~mm}$.

Results - Results showed increased asymmetry from T1 to T2. The average distance increased for whole face (from $0.51 \mathrm{~mm}$ to $0.59 \mathrm{~mm}, \mathrm{p}=0.001$ ), upper face (from $0.41 \mathrm{~mm}$ to $0.49 \mathrm{~mm}$, $\mathrm{p}=0.005$ ), mid-face (from $0.48 \mathrm{~mm}$ to $0.57, \mathrm{p}=0.002$ ) and lower face (from $0.74 \mathrm{~mm}$ to 0.85 $\mathrm{mm}, \mathrm{p}=0.147)$. Facial symmetry percentage decreased for whole face from $61.23 \%$ to $55.38 \%$ $(\mathrm{p}=0.011)$, for upper face from $69.27 \%$ to $62.24 \%(\mathrm{p}=0.005)$ and for mid-face from $62.29 \%$ to $55.63 \%(\mathrm{p}=0.007)$ and for lower face from $43.37 \%$ to $42.19 \%(\mathrm{p}=0.66)$

Conclusion - Facial asymmetry increases from childhood to adulthood in subjects treated for DDH. Orthodontic treatment does not eliminate this asymmetric facial growth. 
Introduction

Developmental dysplasia of the hip (DDH) is the most common musculoskeletal disorder in newborns with an incidence of 1-10/1000. The severity of the condition varies from a mildly shallow acetabulum or "hip socket" to total dislocation of the hip. The primary treatment is to ensure concentric positioning of the femoral head by splinting appliances which requires forced lying on the back for weeks and months. ${ }^{1}$

The etiology of DDH is multifactorial. Breech presentation, female sex, positive family history and left hip side are the most significant risk factors. ${ }^{2,3} \mathrm{DDH}$ is also associated with other congenital postnatal deformities such as plagiocephaly, ${ }^{4}$ scoliosis ${ }^{5}$ and torticollis..${ }^{6,7}$ The reasons for these associations are unclear. Previously in literature, postnatal facial asymmetry has been detected to have association with torticollis, scoliosis ${ }^{8}$ and especially with plagiocephaly. ${ }^{6,9,10}$

3D stereophotography is a novel method in the orthodontic analysis kit. It is an accurate and repeatable tool in the study of facial morphology. ${ }^{11,12}$ As a non-invasive method, it is a good tool for studying facial soft tissue growth changes in children ${ }^{13,14}$ and facial asymmetry. ${ }^{15-17}$ Only few longitudinal 3D studies about facial asymmetry have been made. ${ }^{18,19}$

We have previously found that 8-year-old children treated for DDH had a significantly increased frequency of facial asymmetries ${ }^{15,20}$ and crossbites. ${ }^{21}$ The purpose of this follow-up cohort study is to determine the possible change in facial asymmetry in these subjects from childhood to adolescence using 3D imaging.

Materials and methods

Subjects 
130 newborns born and diagnosed with DDH in Oulu University Hospital during 1997־-2001 were recruited into the previous study in $2007^{15,20,21}$ (T1). The inclusion and exclusion criteria have been described earlier. 60 subjects participated at that time, and all were invited to this follow-up study in 2016 (T2). Approval from the local ethics committee was obtained. The study was registered with the National Clinical Trials register (NCT02431871).

At the first examination (T1), 60 out of 130 neonatally treated subjects participated; of these, 39 (65\%) adolescents (26 females and 13 males) participated in the follow-up (T2). The mean age was 8.1 (SD 1.4) years at $\mathrm{T} 1$ and $16.6(\mathrm{SD} 1.4)$ years at $\mathrm{T} 2$. There were no significant differences between participants and drop-outs in gender, age, crossbite or expected orthodontic treatment. Descriptive statistics of T1 and T2 examination of subjects participating at follow-up are presented in Table 2.

Imaging system

The imaging system used in 2007 (T1) was a portable $3 \mathrm{dMDface}{ }^{\mathrm{TM}}$ System (Atlanta, GA), and at the follow up in 2016 (T2), 3dMDhead ${ }^{\mathrm{TM}}$ System (Atlanta, GA) was used.

Subjects were seated on an adjustable chair at standard distance to center the head optimally, and asked to look forward, maintain natural head position and to keep their face relaxed. The system was calibrated before each imaging session.

Occlusal examination 
All subjects had a clinical dental and occlusal examination including intra-oral photographs performed by one author specialized in orthodontics $(\mathrm{VH})$ in both series. The dental examination included the registration of occlusion according to a modified method of Björk et al. ${ }^{22}$ The sagittal occlusion on both sides was defined by Angles classification. Sagittal occlusion was determined asymmetric if the difference between the right and left side was half a cusp or more. Crossbite was diagnosed if at least one maxillary tooth had buccal cusps that occluded lingually to those of mandibular teeth. The deviation $(\mathrm{mm})$ of dental midline from facial midline was determined both from maxillary tooth and mandibular tooth. Occlusion was determined asymmetric if the deviation of dental midline from facial midline was more than 2 $\mathrm{mm}$, or if sagittal occlusion was asymmetric, or if there was at least one tooth in crossbite. Information on previous orthodontic treatments was gathered from health-care providers.

Image Analysis

Images were processed and analyzed with Rapidform2006 software (Geomagic, Rock Hill, South Carolina). Distinct parts like hair were removed from each image. The position of the faces was standardized as proposed in. ${ }^{23}$ Twenty-one soft tissue landmarks ${ }^{24}$ were identified by one author. (Figure 1, Table 1) Subjects' facial surfaces from both time points were scaled to the average Procrustes size (Frobenius norm of landmark matrix, in which landmark coordinates are in rows).

Measurements 
The facial image was divided into three parts: upper face (above endocanthion plane), mid face (between the endocanthion and cheilion plane (labial commissure)) and lower face (under the cheilion plane). (Figure 1, Table 1) A mirrored facial surface was formed mirroring facial parts across the sagittal plane (YZ plane), and these two surfaces were superimposed using the bestfit technique.

The average distance $(\mathrm{mm})$ was calculated between the original and mirrored face (Figure 2). The symmetry percentage (\%) was calculated as the face area where the distance between the original face and the mirrored surface does not exceed $0.5 \mathrm{~mm}$. The chosen $0.5 \mathrm{~mm}$ limit to symmetry percentage has also been used in previous studies. ${ }^{18,25}$

Angles ExR-exL-pg and ExL-exR-pg (formed by a line connecting the exocanthions and pogonion) were measured both in 3D space and also for their orthogonal projections onto the coronal plane (XY plane). Expg diff (Absolute value of difference of exRexL-pg \& exL-exR-pg) was calculated from these angles.

Position standardizing, scaling and computation of the parameters have been automated with a set of in-house VBA (Visual Basic for Applications) subroutines developed for Rapidform.

Statistical Analysis

IBM SPSS 24 statistics software was used. Normality was assessed using Shapiro-Wilk normality test. Paired-Samples T-test was used to analyze differences between T1 and T2 for normally distributed parameters and Wilcoxon signed-rank test was used for non-normal data. Independent-Samples t-test or Mann-Whitney test was used to test the difference of the changes over time between the following groups: male and female, children with previous orthodontic 
treatment and non-treated subjects, or children with or without asymmetric occlusion. A P value of $<0.05$ was considered significant.

Results

The degree of asymmetry measured as average distance between the original face and mirror face increased from time point T1 to T2 (Table 3). The average distance increased statistically significantly for whole face from $0.51 \mathrm{~mm}$ to $0.59 \mathrm{~mm}$ ( $\mathrm{p}=0.001$ ), for upper face from $0.41 \mathrm{~mm}$ to $0.49 \mathrm{~mm}(\mathrm{p}=0.005)$ and for mid-face from $0.48 \mathrm{~mm}$ to $0.57(\mathrm{p}=0.002)$. Correspondingly, facial symmetry percentage decreased statistically significantly for whole face from $61.23 \%$ to $55.38 \%(\mathrm{p}=0.011)$, for upper face from $69.27 \%$ to $62.24 \%(\mathrm{p}=0.005)$ and for mid-face from $62.29 \%$ to $55.63 \%(\mathrm{p}=0.007)$. (Table 3$)$.

There was no statistically significant change in symmetry parameters for lower face. The average distance increased for lower face from $0.74 \mathrm{~mm}$ to $0.85 \mathrm{~mm}(\mathrm{p}=0.147)$ and facial symmetry percentage decreased for lower face from $43.37 \%$ to $42.19 \%(\mathrm{p}=0.655)$ (Table $3)$.

There was a statistically significant difference $(\mathrm{p}<0.001)$ in the increase of the following angular parameters: exR-exL-pg, exL-exR-pg and the difference in the angles exRexL-pg and exL-exR-pg (ex-pg diff). A statistically significant difference was found both in 3Dspace $(\mathrm{p}=0.023)$ and in XY-plane $(\mathrm{p}=0.015)($ Table 3$)$.

At T2, 29 out of 39 (74.4\%) participants had orthodontic treatment, beginning on average at 9.5 (SD 2.3) years and lasting on average 4.0 (SD 2.6) years. 13 (33.3\%) participants had already begun their orthodontic treatment before $\mathrm{T} 1$ and only one of them had finished orthodontic treatment before $\mathrm{T} 1$. All others continued their treatment between the T1 and T2 
time points (Table 2). 15 out of 29 subjects had had orthodontic treatment for correction of sagittal relationship, while 23 out of 29 had needed it for correction of transversal relationship or crowding. A total of 9 children had been treated to repair both sagittal and transverse relationships.

The subjects who had orthodontic treatment had a lower symmetry percentage, indicating higher asymmetry, at mid-face (59.6\%) at initial scanning (T1) compared to subjects without any orthodontic treatment at all (symmetry percentage $70,2 \%)(\mathrm{p}=0.03)$. Similarly, the subjects who had orthodontic treatment had a larger average distance at mid face $(0.51 \mathrm{~mm})$ at $\mathrm{T} 1$ compared to the subjects without orthodontic treatment $(0.41 \mathrm{~mm})(\mathrm{p}=0.018)$. During follow-up, from time point $\mathrm{T} 1$ to $\mathrm{T} 2$, the facial symmetry percentage in the mid-face area decreased more in the group without orthodontic treatment (from $70.2 \%$ to $55.1 \%$ ) compared to the treated group (from $59.6 \%$ to $55.8 \%$ ), $\mathrm{p}=0.032$.

The difference in angles exR-exL-pg and exL-exR-pg (ex-pg diff), which represents deviation of the chin, was larger at $\mathrm{T} 2$ in the subjects who had had asymmetric occlusion at $\mathrm{T} 1$ compared to the subjects without asymmetric occlusion. The results were statistically significant both in $3 \mathrm{D}$ space $\left(2.1^{\circ}\right.$ and $1.3^{\circ}$, respectively, $\left.\mathrm{p}=0.035\right)$ and in coronal plane $\left(2.2^{\circ}\right.$ and $1.3^{\circ}$, respectively, $\left.\mathrm{p}=0.013\right)$.

There was no statistically significant difference in symmetry parameters between the genders.

Discussion

This study examines the change in facial asymmetry among subjects with a history of developmental dysplasia of the hip (DDH). To our knowledge, there are no previous 
longitudinal studies of facial asymmetry among subjects treated for DDH. Our earlier studies showed that children with history of DDH have more facial and occlusal asymmetry than control group children..$^{20,21}$

In the present series, the average distance between the original and mirrored facial surfaces increased and facial symmetry percentage decreased in every area of the face between $\mathrm{T} 1$ and $\mathrm{T} 2$ time points, indicating that the face became more asymmetric in the patients treated for DDH. The difference was statistically significant for all other parts of the face except lower jaw. The increase of the angles exL-exR-pg or exR-exL-pg over time indicates normal vertical facial growth. Although facial symmetry percentage or average distance did not change statistically significantly in the lower jaw, the increase in the difference in angles exR-exL-pg and exL-exR-pg (ex-pg diff), representing increase in the deviation of the chin, was statistically significant.

According to the earlier three-dimensional studies, mandibular asymmetry doesn't significantly change over time,${ }^{18}$ although the mandibular bone shows some fluctuating asymmetry during normal facial growth. ${ }^{26}$ Djordjevic et al. ${ }^{18}$ found among healthy adolescents of the same ethnic origin and corresponding age group that facial asymmetry did not change significantly over time in any area of the face. In the present series, the results show increased asymmetry, which points to the late growth effect of DDH on facial growth.

It is known that DDH is associated with different congenital postnatal deformities. ${ }^{5-7}$ In our former cross-sectional 3D imaging study of the same population, Tolleson et al. found facial asymmetry in childhood after treatment of DDH. ${ }^{15}$ The difference was significant in comparison to controls. ${ }^{20}$ There is a clear genetic predisposition to DDH with a 12-fold increased risk for first degree relatives. ${ }^{27}$ Various susceptibility genes to DDH have been identified. Most of these are involved in chondrogenesis and joint formation, ${ }^{28}$ and the association of DDH and facial asymmetry could, at least partly, be explained by genetic factors. 
It is known that supine sleeping, infant inactivity and little prone time are risk factors of deformational plagiocephaly. ${ }^{29}$ In a three-dimensional-imaging study, Aarnivala et al. found that providing the parents of newborns with instructions on their infant's sleeping position significantly reduced the prevalence and severity of developmental plagiocephaly among newborns. ${ }^{30}$ During long-lasting DDH treatment the baby is forced into supine position. It can be speculated whether this could explain the earlier found higher incidence of plagiocephaly ${ }^{5}$ and torticollis ${ }^{7}$ among treated children, and further induce mandibular asymmetry by rotation of the cranial base and anterior position of the temporomandibular joint. ${ }^{10}$

Harila et al. found earlier among this study population that children aged 5-10 years and with history of DDH have high prevalence (30\%) of crossbite compared to controls $(9 \%) .{ }^{21}$ In this follow-up, crossbites decreased from $30.8 \%$ at $\mathrm{T} 1$ to $7.7 \%$ at $\mathrm{T} 2$ after orthodontic treatment of the follow-up cohort. However, the prevalence of orthodontic treatment was higher in this study (74.4\%) than reported in unselected Finnish population $(52.4 \%) \cdot{ }^{31}$ It is remarkable that orthodontic treatment succeeded in correcting the majority of crossbites without significant correction of facial asymmetry. This contradicts the findings of a 3D longitudinal study by Primozic et al., ${ }^{25}$ where early crossbite correction improved facial asymmetry in a 30-month follow-up of schoolchildren.

In the present study, facial asymmetry in mid-face at $\mathrm{T} 1$ was associated with the need of orthodontic treatment. Nevertheless, the facial symmetry percentage in mid-face diminished from $\mathrm{T} 1$ to $\mathrm{T} 2$ particularly in the group without orthodontic treatment, indicating the asymmetrical growth tendency in the whole group. Also, the increase in the difference in angles exR-exL-pg and exL-exR-pg (T2) among subjects with history of asymmetric occlusion (T1) indicates persisting asymmetric growth potential of the lower jaw.

A major strength in this series was that facial surfaces at $\mathrm{T} 1$ and $\mathrm{T} 2$ were not compared to one another directly: facial images were only compared to mirrored ones. To 
minimize any effect of facial growth that could modify the result, the size of the facial surfaces of subjects taken at different time points (T1 and $\mathrm{T} 2)$ were scaled to equal.

One possible source of error when analyzing images is that pogonion (pg) in the lower jaw is probably the most unstable landmark. Furthermore, only two of the Farkas landmarks are in the lower part of the face, making the result uncertain with respect to the lower jaw.

According to this study, the previously found asymmetric tendency continues during the pubertal growth despite orthodontic treatment in subjects treated for DDH. Therefore, we consider that special attention should be paid to diagnose and treat orthodontic problems among DDH patients as early as possible to prevent the development of asymmetric discrepancies. In further research, the focus should be on how to decrease and prevent development of facial asymmetry at an early age in these subjects. Studies are needed also concerning the genetic background of the association of congenital deformations and facial asymmetry.

Conclusion

Facial asymmetry increases during puberty among subjects with developmental dysplasia of the hip. Orthodontic treatment does not eliminate this asymmetric facial growth.

\section{Conflict of interest}

The authors declare no conflicts of interest. 


\section{References}

1. Shaw BA, Segal LS. Evaluation and referral for developmental dysplasia of the hip in infants. Pediatrics. 2016;138.

2. de Hundt M, Vlemmix F, Bais JMJ, et al. Risk factors for developmental dysplasia of the hip: A meta-analysis. Eur J Obstet Gynecol Reprod Biol. 2012;165:8-17.

3. Ortiz-Neira CL, Paolucci EO, Donnon T. A meta-analysis of common risk factors associated with the diagnosis of developmental dysplasia of the hip in newborns. Eur J Radiol. $2012 ; 81: 344$

4. Lambeek AF, De Hundt M, Vlemmix F, et al. Risk of developmental dysplasia of the hip in breech presentation: The effect of successful external cephalic version. BJOG. 2013;120:607612.

5. Watson GH. Relation between side of plagiocephaly, dislocation of hip, scoliosis, bat ears, and sternomastoid tumours. Arch Dis Child. 1971;46:203-210.

6. van Vlimmeren LA, Helders PJ, van Adrichem LN, Engelbert RH. Diagnostic strategies for the evaluation of asymmetry in infancy-a review. Eur J Pediatr. 2004;163:185-191.

7. von Heideken J, Green DW, Burke SW, et al. The relationship between developmental dysplasia of the hip and congenital muscular torticollis. J Pediatr Orthop. 2006;26:805-808.

8. Pirttiniemi PM. Associations of mandibular and facial asymmetries--a review. Am J Orthod Dentofacial Orthop. 1994;106:191-200.

9. Moon IY, Lim SY, Oh KS. Analysis of facial asymmetry in deformational plagiocephaly using three-dimensional computed tomographic review. Arch Craniofac Surg. 2014;15:109-116. 
10. St John D, Mulliken JB, Kaban LB, Padwa BL. Anthropometric analysis of mandibular asymmetry in infants with deformational posterior plagiocephaly. J Oral Maxillofac Surg. 2002;60:873-877.

11. Kau CH, Richmond S, Zhurov AI, et al. Reliability of measuring facial morphology with a 3-dimensional laser scanning system. Am J Orthod Dentofacial Orthop. 2005;128:424-430.

12. Aldridge K, Boyadjiev SA, Capone GT, DeLeon VB, Richtsmeier JT. Precision and error of three-dimensional phenotypic measures acquired from 3dMD photogrammetric images. Am J Med Genet A. 2005;138A:247-253.

13. Kau CH, Richmond S. Three-dimensional analysis of facial morphology surface changes in untreated children from 12 to 14 years of age. Am J Orthod Dentofacial Orthop. 2008;134:751760.

14. Primozic J, Perinetti G, Contardo L, Ovsenik M. Facial soft tissue changes during the prepubertal and pubertal growth phase: A mixed longitudinal laser-scanning study. Eur J Orthod. 2017;39:52-60.

15. Tolleson SR, Kau CH, Lee RP, et al. 3-D analysis of facial asymmetry in children with hip dysplasia. Angle Orthod. 2010;80:519-524.

16. White JE, Ayoub AF, Hosey MT, et al. Three-dimensional facial characteristics of caucasian infants without cleft and correlation with body measurements. Cleft Palate Craniofac J. 2004;41:593-602.

17. Djordjevic J, Toma AM, Zhurov AI, Richmond S. Three-dimensional quantification of facial symmetry in adolescents using laser surface scanning. Eur J Orthod. 2014;36:125-132.

18. Djordjevic J, Pirttiniemi P, Harila V, et al. Three-dimensional longitudinal assessment of facial symmetry in adolescents. Eur J Orthod. 2013;35:143-151. 
19. Primozic J, Perinetti G, Zhurov A, Richmond S, Ovsenik M. Assessment of facial asymmetry in growing subjects with a three-dimensional laser scanning system. Orthod Craniofac Res. 2012;15:237-244.

20. Hanis SB, Kau CH, Souccar NM, et al. Facial morphology of finnish children with and without developmental hip dysplasia using 3D facial templates. Orthod Craniofac Res. 2010;13:229-237.

21. Harila V, Valkama M, Sato K, et al. Occlusal asymmetries in children with congenital hip dislocation. Eur J Orthod. 2012;34:307-311.

22. Björk A, Krebs A, Solow B. A method for epidemiological registration of malocculusion. Acta Odontologica Scandinavica. 1964;22:27-41.

23. Zhurov A, Richmond S, Kau CH, Toma A. Averaging facial images. In: Three-dimensional imaging for orthodontics and maxillofacial surgery. John Wiley \& Sons, Ltd.; 2010:126-144.

24. Farkas L, ed. Anthropometry of the head and face. New York: Raven Press; 1994.

25. Primozic J, Richmond S, Kau CH, Zhurov A, Ovsenik M. Three-dimensional evaluation of early crossbite correction: A longitudinal study. Eur J Orthod. 2013;35:7-13.

26. Liukkonen M, Sillanmäki L, Peltomäki T. Mandibular asymmetry in healthy children. Acta Odontol Scand. 2005;63:168-172.

27. Stevenson DA, Mineau G, Kerber RA, Viskochil DH, Schaefer C, Roach JW. Familial predisposition to developmental dysplasia of the hip. J Pediatr Orthop. 2009;29:463-466.

28. Basit S, Hannan MA, Khoshhal KI. Developmental dysplasia of the hip: Usefulness of next generation genomic tools for characterizing the underlying genes - a mini review. Clin Genet. 2016;90:16-20 
29. Hutchison BL, Thompson JMD, Mitchell EA. Determinants of nonsynostotic plagiocephaly: A case-control study. Pediatrics. 2003;112:e316.

30. Aarnivala H, Vuollo V, Harila V, Heikkinen T, Pirttiniemi P, Valkama AM. Preventing deformational plagiocephaly through parent guidance: A randomized, controlled trial. Eur J Pediatr. 2015;174:1197-1208.

31. Svedström-Oristo A, Pietilä T, Pietilä I, Tolvanen M, Varrela J, Alanen P. An analysis of residual orthodontic treatment need in municipal health centres. Eur J Orthod. 2015;37:398402.

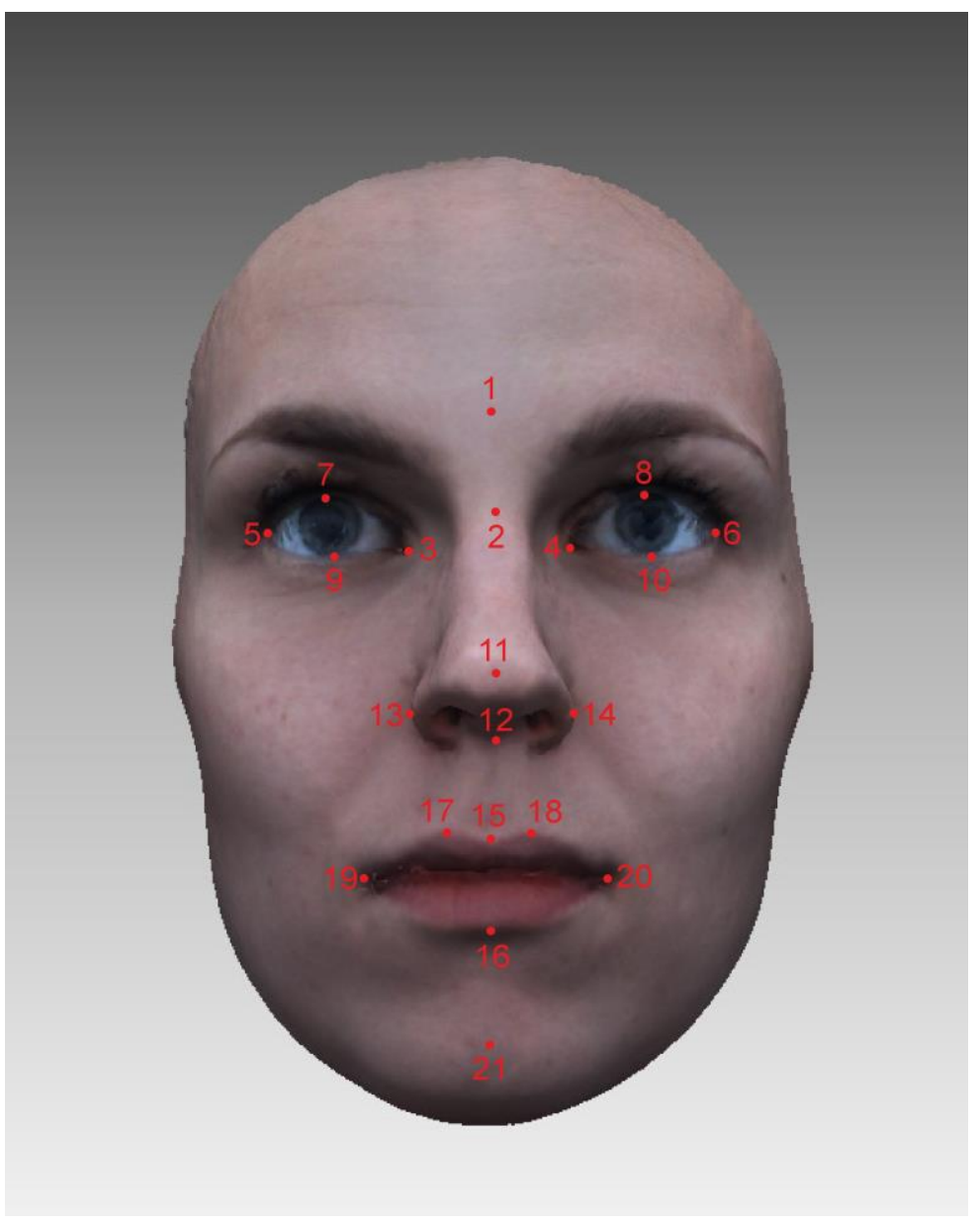

Figure 1: The Farkas soft-tissue landmarks 


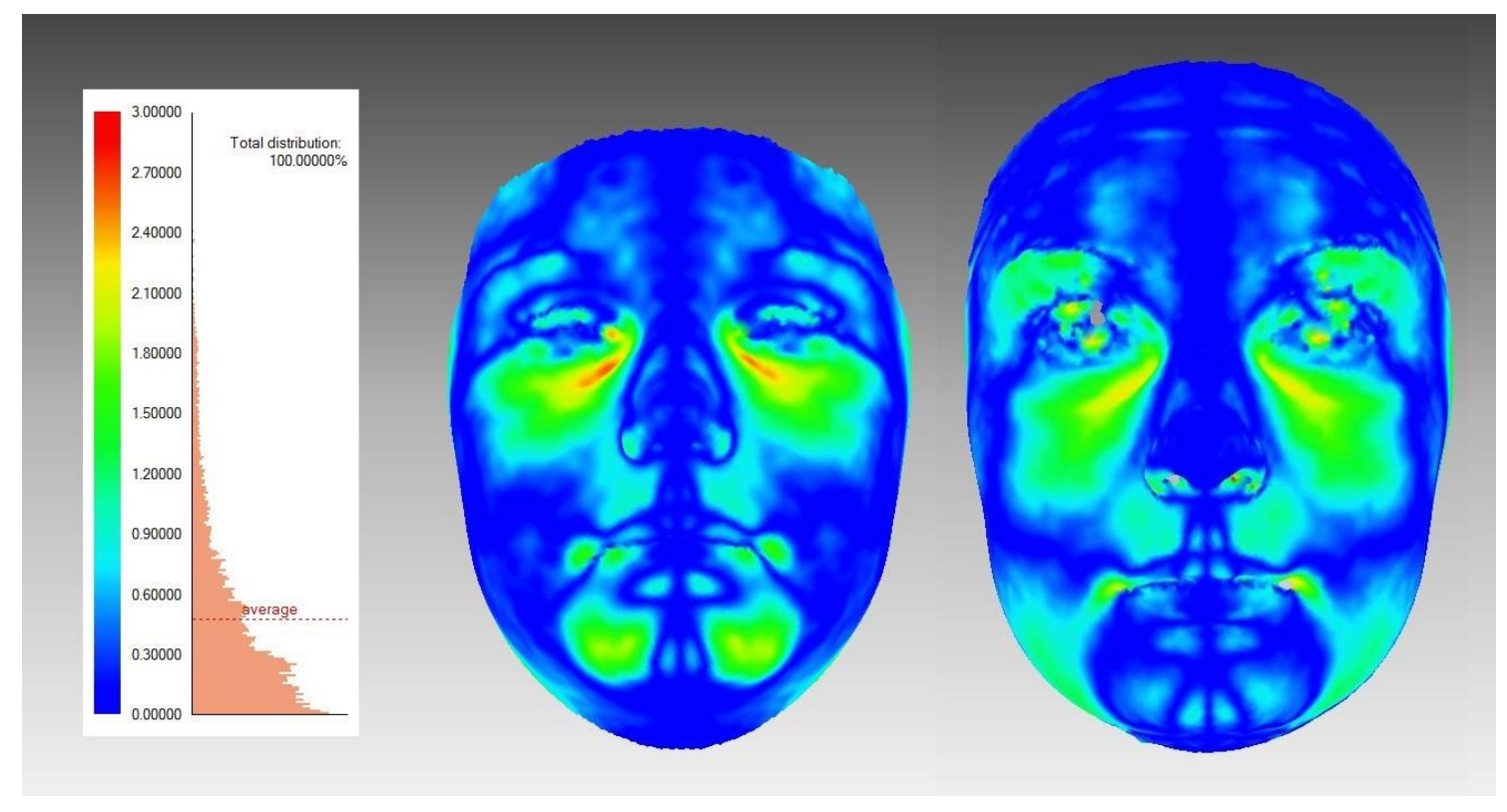

Figure 2:

An example of facial symmetry of one participant at the first examination (T1) and at the followup (T2) illustrated by color maps. The colors of the face regions indicate the deviation ( $\mathrm{mm}$ ) between the original and the mirrored facial surface (dark blue 0.0-0.4mm, turquoise 0.5-1.1, light green 1.2-2.0, yellow 2.1-2.3, orange, 2.4-2.6, red 2.7-3.0) 
Table 1: The Farkas landmarks

\begin{tabular}{|c|c|c|}
\hline Landmarks & Abbreviation & Definition \\
\hline 1 Glabella & g & The most prominent midpoint between the eyebrows \\
\hline 2 Nasion & $\mathrm{n}$ & $\begin{array}{l}\text { Midline point between the nasal root and nasofrontal suture, above the line } \\
\text { that connects the two inner canthi }\end{array}$ \\
\hline 3,4 Endocanthion & en & The point at the inner commissure of the eye fissure \\
\hline 5,6 Exocanthion & ex & The point at the outer commissure of the eye fissure \\
\hline 7,8 Pulpabrale superius & ps & The highest point in the midportion of the free margin of each upper eyelid \\
\hline 9,10 Pulpabrale inferius & pi & The lowest point in the midportion of the free margin of each lower eyelid \\
\hline 11 Pronasale & prn & The most protruded point of apex nasi \\
\hline 12 Subnasale & sn & $\begin{array}{l}\text { The midpoint of the angle at the columella base where the lower border of } \\
\text { the nasal septum and the surface of the upper lip meet }\end{array}$ \\
\hline 13,14 Alare & al & The most lateral point on each alar contour \\
\hline 15 Labiale superius & Is & The midpoint of the upper vermilion line \\
\hline 16 Labiale inferius & li & The midpoint of the lower vermilion line \\
\hline 17, 18 Christa philtri & $\mathrm{cph}$ & The point on each elevated margin of the philtrum above the vermilion line \\
\hline 19,20 Cheilion & ch & The point at each labial commissure \\
\hline 21 Pogonion & $\mathrm{pg}$ & The most prominent midpoint of the chin \\
\hline
\end{tabular}


Table 2: The baseline (T1) characteristics of participants and dropouts and Follow-up characteristics of the participants at T2

${ }^{1}$ Pearsons Chi-Square was used for dichotomous variables and independent samples T-test for continuous variables

${ }^{2}$ No statistical test was used due to small expected count++

\begin{tabular}{|c|c|c|c|c|c|c|c|c|c|c|c|c|c|c|c|c|}
\hline \multirow[t]{2}{*}{ Characteristics } & \multicolumn{11}{|c|}{$\mathrm{T1}(\mathrm{N}=60)$} & \multicolumn{5}{|c|}{ T2 $(\mathrm{N}=39)$} \\
\hline & \multicolumn{5}{|c|}{ Participants at follow-up $(\mathrm{N}=39)$} & \multicolumn{5}{|c|}{ Drop-outs $(\mathrm{N}=21)$} & \multirow{2}{*}{$\begin{array}{c}\mathrm{P} \\
\text { Value }^{1} \\
\mathrm{p}\end{array}$} & & & & & \\
\hline & $\mathrm{N}$ & $\%$ & Mean & SD & Range & $\mathrm{N}$ & $\%$ & Mean & SD & Range & & $\mathrm{N}$ & $\%$ & Mean & SD & Range \\
\hline Male & 26 & 66.7 & & & & 14 & 66.7 & & & & 1.000 & & & & & \\
\hline Female & 13 & 33.3 & & & & 7 & 33.3 & & & & 1.000 & & & & & \\
\hline Age & & & 8.15 & 1.4 & $5.9-10.4$ & & & 7.95 & 1.45 & $5.8-10.3$ & 0.785 & & & 16.6 & 1.4 & 14.3-18.9 \\
\hline Height & & & 129.6 & 10.8 & $107.9-154.9$ & & & 130.9 & 10.0 & $112.1-150.0$ & 0.660 & & & 168.6 & 6.9 & $157.0-188.0$ \\
\hline Weight & & & 29.5 & 9.1 & $16.8-56.6$ & & & 33.3 & 10.8 & 19.4-57.0 & 0.154 & & & 61.5 & 11.2 & $42.1-90.5$ \\
\hline Head circumference & & & 53.8 & 1.6 & $50.8-57.0$ & & & 54.41 & 1.28 & $51.9-57.0$ & 0.114 & & & 54.8 & 2.3 & $52.5-57.9$ \\
\hline Crossbite & 12 & 30.8 & & & & 3 & 14.3 & & & & 0.160 & 3 & 7.7 & & & \\
\hline $\begin{array}{l}\text { Deviation of dental midline } \\
\text { of facial midline }>2 \mathrm{~mm}\end{array}$ & 4 & 10.3 & & & & 0 & 0.0 & & & & $-{ }^{2}$ & 5 & 12.8 & & & \\
\hline Asymmetric Occlusion & 14 & 38.9 & & & & 5 & 23.8 & & & & 0.337 & 15 & 38.5 & & & \\
\hline Asymmetric Angle & 5 & 12.8 & & & & 3 & 14.3 & & & & $-{ }^{2}$ & 9 & 23.1 & & & \\
\hline $\begin{array}{l}\text { Orthodontic treatment on- } \\
\text { going or previously }\end{array}$ & 13 & 33.3 & & & & 5 & 23.8 & & & & 0.198 & 29 & 74.4 & & & \\
\hline
\end{tabular}


Table 3: The average distance, facial symmetry percentage and facial angles at the first examination (T1) and at follow-up screening (T2)

${ }^{1}=$ paired $T$-test was used, ${ }^{2}=$ Wilcoxon test was used.

\begin{tabular}{|c|c|c|c|c|c|c|c|c|c|c|c|}
\hline & $\mathrm{T} 1(\mathrm{~N}=39)$ & & & $\mathrm{T} 2(\mathrm{~N}=39)$ & & & T1-T2 & & & & \\
\hline & Mean (SD) & Median & Range & Mean (SD) & Median & Range & Mean (SD) & Median & Range & $95 \%$ Confidence & $\mathrm{p}$ \\
\hline Part of the face & Average dist. ( & & & Average dist. ( & & & Average dist. & $\mathrm{mm})$ & & & \\
\hline Whole Face & $0.51(0.15)$ & 0.48 & $(0.41-0.56)$ & $0.59(0.18)$ & 0.56 & $(0.46-0.71)$ & $-0.08(0.16)$ & -0.09 & $(-0.16-0.00)$ & $(-0.14--0.03)$ & $0.001^{2}$ \\
\hline Upper Face & $0.41(0.14)$ & 0.41 & $(0.31-0.50)$ & $0.49(0.18)$ & 0.43 & $(0.36-0.58)$ & $-0.07(0.15)$ & -0.06 & $(-0.19-0.01)$ & $(-0.12--0.03)$ & $0.005^{2}$ \\
\hline Mid face & $0.48(0.16)$ & 0.48 & $(0.35-0.52)$ & $0.57(0.18)$ & 0.53 & $(0.44-0.66)$ & $-0.08(0.15)$ & -0.09 & $(-0.22-0.03)$ & $(-0.13--0.03)$ & $0.002^{2}$ \\
\hline Lower face & $0.74(0.30)$ & 0.69 & $(0.45-0.98)$ & $0.85(0.42)$ & 0.72 & $(0.56-1.03)$ & $-0.11(0.47)$ & -0.06 & $(-0.38-0.14)$ & $(-0.26-0.04)$ & $0.147^{2}$ \\
\hline & Symmetry (\%) & & & Symmetry (\%) & & & Symmetry (\% & & & & \\
\hline Whole Face & $61.23(12.37)$ & 61.80 & (54.46-70.74) & $55.38(12.53)$ & 55.48 & (47.36-63.09) & $5.86(13.69)$ & 7.12 & $(-3.40-13.61)$ & $(1.42-10.30)$ & $0.011^{1}$ \\
\hline Upper Face & $69.27(15.07)$ & 70.36 & $(59.42-79.74)$ & $62.24(15.26)$ & 66.07 & (51.98-71.43) & $7.03(14.75)$ & 9.27 & $(-1.49-18.03)$ & $(2.25-11.82)$ & $0.005^{1}$ \\
\hline Mid face & 62.29 (13.77) & 61.34 & $(53.17-74.36)$ & $55.63(13.55)$ & 55.04 & $(48.35-67.33)$ & $6.66(14.67)$ & 5.17 & $(-0.33-16.75)$ & $(1.91-11.42)$ & $0.007^{1}$ \\
\hline Lower face & $43.37(20.84)$ & 40.01 & $(24.63-64.12)$ & 42.19 (20.13) & 43.69 & $(21.95-53.92)$ & $1.18(27.38)$ & 3.82 & $(-17.42-17.08)$ & $(-7.70-10.05)$ & $0.655^{2}$ \\
\hline Angles & Angles $\left({ }^{\circ}\right)$ & & & Angles $\left({ }^{\circ}\right)$ & & & Angles $\left({ }^{\circ}\right)$ & & & & \\
\hline exR-exL-pg. 3D & 63.89 (1.62) & 64.12 & $(62.58-65.31)$ & $65.05(1.62)$ & 65.27 & (63.71-65.88) & $-1.17(1.24)$ & -1.14 & $(-2.20--0.19)$ & $(-1.57--0.76)$ & $<0.001^{1}$ \\
\hline exR-exL-pg. XY & $63.00(1.70)$ & 63.01 & $(61.59-64.36)$ & $64.30(1.68)$ & 64.54 & $(62.90-65.23)$ & $-1.30(1.24)$ & -1.25 & $(-2.29--0.40)$ & $(-1.70--0.90)$ & $<0.001^{1}$ \\
\hline exL-exR-pg. 3D & $63.60(1.66)$ & 63.60 & $(62.67-64.60)$ & $66.00(1.60)$ & 65.84 & (64.98-67.03) & $-2.40(1.29)$ & -2.37 & $(-3.47--1.32)$ & $(-2.82--1.98)$ & $<0.001^{1}$ \\
\hline exL-exR-pg. XY & $62.75(1.78)$ & 62.62 & $(61.66-63.70)$ & $65.23(1.79)$ & 65.14 & $(64.30-66.45)$ & $-2.48(1.45)$ & -2.48 & $(-3.57--1.56)$ & $(-2.95--2.01)$ & $<0.001^{1}$ \\
\hline & Difference of a & gles & & & & & & & & & \\
\hline expg diff. 3D & $1.10(0.98)$ & 0.95 & $(0.27-1.78)$ & 1.57 (1.19) & 1.42 & $(0.54-2.23)$ & $-0.47(1.51)$ & -0.35 & $(-1.22-0.11)$ & $(-0.95-0.02)$ & $0.023^{2}$ \\
\hline expg diff. XY & $1.04(1.01)$ & 0.78 & $(0.30-1.68)$ & $1.60(1.15)$ & 1.49 & $(0.85-1.89)$ & $-0.56(1.53)$ & -0.58 & $(-1.60-0.17)$ & $(-1.05--0.06)$ & $0.015^{2}$ \\
\hline
\end{tabular}


